\title{
Photocatalytic Hydrogen Evolution from Water Using Fluorene and Dibenzothiophene Sulfone-Conjugated Microporous and Linear Polymers
}

\author{
Reiner Sebastian Sprick, ${ }^{* \dagger \odot}$ Yang Bai, ${ }^{\dagger}$ Anne A. Y. Guilbert, ${ }^{\ddagger \odot}$ Mohamed Zbiri, \\ Catherine M. Aitchison, ${ }^{\dagger}$ Liam Wilbraham, ${ }^{\|}$Yong Yan, ${ }^{\dagger}$ Duncan J. Woods, ${ }^{\dagger}$ \\ Martijn A. Zwijnenburg,
}

${ }^{\dagger}$ Department of Chemistry and Materials Innovation Factory, University of Liverpool, Crown Street, Liverpool L69 7ZD, U.K.

${ }^{\ddagger}$ Department of Physics and Centre for Plastic Electronics, Imperial College London, Prince Consort Road, London SW7 2AZ, U.K.

${ }^{\S}$ Institut Laue-Langevin, 71 Avenue des Martyrs, Grenoble Cedex 9 38042, France

"Department of Chemistry, University College London, 20 Gordon Street, London WC1H 0AJ, U.K.

\author{
Supporting Information
}

ABSTRACT: Three series of conjugated microporous polymers (CMPs) were studied as photocatalysts for hydrogen production from water using a sacrificial hole scavenger. In all cases, dibenzo $[b, d]$ thiophene sulfone polymers outperformed their fluorene analogues. A porous network, SCMP3, showed the highest hydrogen evolution rates of 6076 $\mu \mathrm{mol} \mathrm{h} \mathrm{g}^{-1}(\lambda>295 \mathrm{~nm})$ and $3106 \mu \mathrm{mol} \mathrm{h}^{-1} \mathrm{~g}^{-1}(\lambda>420$ $\mathrm{nm})$, with an external quantum efficiency of $13.2 \%$ at $420 \mathrm{~nm}$. S-CMP3 outperforms its linear structural analogue, P35, whereas in other cases, nonporous linear polymers are superior to equivalent porous networks. This suggests that microporosity might be beneficial for sacrificial photocatalytic
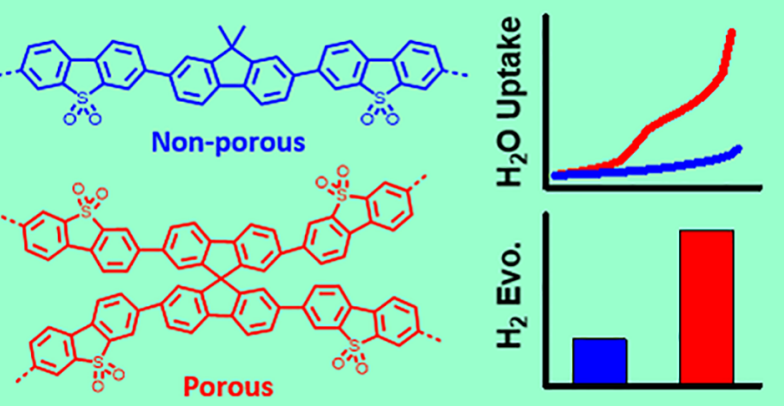
hydrogen evolution, if suitable linkers are used that do not limit charge transport and the material can be wetted by water as studied here by water sorption and quasi-elastic neutron scattering.

\section{INTRODUCTION}

The use of hydrogen as an energy carrier has the potential to radically reduce greenhouse gas emissions from hydrocarbon combustion but, at the moment, most hydrogen is produced by methane steam reforming. Photocatalytic water splitting has the potential to produce hydrogen from water with oxygen as the only side product. Inorganic oxide materials such as $\mathrm{TiO}_{2}$, $\mathrm{WO}_{3}$, and $\mathrm{SrTiO}_{3}$ have been studied for hydrogen and oxygen production via oxidative or reductive half reactions; based on these half reactions, overall water splitting has been achieved, ${ }^{1}$ for example, via Z-schemes ${ }^{2,3}$ and heterojunctions. ${ }^{4}$ To be scalable, photocatalysts should be based on earth-abundant and nontoxic elements. Recently, organic photocatalysts have become the subject of much research, prompted in large part by a study on carbon nitride published in $2009 .^{5-8}$ Other organic material classes such as conjugated polymers, ${ }^{9-16}$ conjugated microporous polymers (CMPs), ${ }^{17-25}$ covalent triazine-based frameworks (CTFs), ${ }^{26-34}$ and covalent organic frameworks $^{35-39}$ have also been explored. Copolymerization with a range of comonomers ${ }^{9,12,40-45}$ and reducing the size of photocatalyst particles have been found to enhance the activity, ${ }^{46-48}$ giving promising results, especially for the hydrogen-evolving half reaction. However, the large structural diversity that is available makes it challenging to formulate simple structure-property relationships.

In particular, we do not yet understand the complex interplay between optical gap, charge mobility, excited state lifetime, and other properties such as particle size, porosity, and surface hydrophilicity. This is compounded by the fact that there are relatively few mechanistic studies for these materials, and it is often unclear whether these polymers act as light absorbers, as catalysts, or as both. Previously, we $\mathrm{w}^{12}$ and others $^{22-24}$ have reported nonporous linear polymers with sacrificial hydrogen evolution rates (HERs) that exceed those that we reported for porous CMPs. This suggested that microporosity may not be essential for good photocatalytic activity-or perhaps even that porosity and linkers that are used might introduce limitations, perhaps by reducing charge carrier mobilities. Here, we test this by comparing a range of porous network polymers and nonporous linear polymers based on either fluorene or dibenzo $[b, d]$ thiophene units copolymerized with one of six different linkers. We investigate

Received: July 5, 2018

Revised: December 17, 2018

Published: December 17, 2018 
the photocatalytic activity for hydrogen evolution from water under sacrificial conditions and show that porous conjugated polymers can outperform linear analogues significantly, although not for all monomer combinations.

\section{RESULTS AND DISCUSSION}

All materials were synthesized using $\operatorname{Pd}(0)$-catalyzed SuzukiMiyaura polycondensation of diboronic acids/diboronic acid ester-functionalized monomers with bromo-functionalized comonomers (Figure 1, see the Supporting Information for

\section{Microporous Networks}
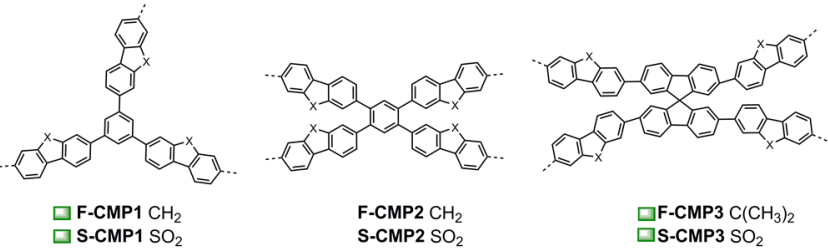

Non-porous Linear Polymers

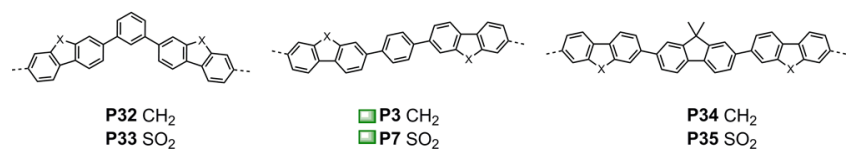

Figure 1. Structures of the polymers used in this study. The linear polymers (second row) contain monomers that mirror the linkers in the CMP networks (first row). For each pairing, the photocatalyst showing higher performance under visible light (CMP or nonporous/ low porosity linear polymer) is marked with a green square, see also Table 1.

synthetic details). ${ }^{12,17}$ The materials were insoluble in organic solvents and in water, and they were characterized by Fourier transform infrared spectroscopy (FT-IR) and elemental analysis (see the Supporting Information). Thermogravimetric analysis (TGA) indicated that the polymers were thermally stable in air up to $300{ }^{\circ} \mathrm{C}$ (Figure S3).

Scanning electron microscopy (SEM) images of the CMPs show that the CMPs all consist of larger aggregates and the linear polymers showed morphologies that were similar to the CMPs. Powder X-ray diffraction (PXRD) showed that the CMP materials and the 1,3-benzene-co-fluorene polymer, P32, were amorphous, whereas $\mathrm{P} 3, \mathrm{P} 7,^{12}$ and $\mathrm{P} 33-\mathrm{P} 35$ were semicrystalline (Figures S4 and S5). Nitrogen sorption isotherms were measured at $77 \mathrm{~K}$ for all materials and apparent Brunauer-Emmett-Teller $\left(\mathrm{SA}_{\mathrm{BET}}\right)$ surface areas were calculated based on the adsorption isotherms (Figures S6-S12). The fluorene derivatives F-CMP1 and F-CMP2 had low apparent surface areas $\left(62\right.$ and $\left.51 \mathrm{~m}^{2} \mathrm{~g}^{-1}\right)$ compared to their dibenzo $[b, d]$ thiophene sulfone analogues, S-CMP1 and S-CMP2 (508 and $560 \mathrm{~m}^{2} \mathrm{~g}^{-1}$ ), whereas F-CMP3 showed a larger surface area than S-CMP3 (596 vs $\left.431 \mathrm{~m}^{2} \mathrm{~g}^{-1}\right)$. The linear polymers all had low surface areas $\left(<70 \mathrm{~m}^{2} \mathrm{~g}^{-1}\right)$, except for the dibenzo[ $[b, d]$ thiophene sulfone containing polymers P33 and P35, which had modest BET surface areas of 192 and $114 \mathrm{~m}^{2} \mathrm{~g}^{-1}$, respectively. It is possible that the rigid polymer P33, with its 1,3-substituted linker, is a polymer of intrinsic microporosity as its adsorption isotherm shows a small micropore step at low relative pressures (Figure S10).

UV/visible absorption spectra were measured for all materials in the solid state (Figures 2a and S21-S30). As expected, the 1,3-linked polymers P32 and P33 have the most blue-shifted absorption onset with optical gaps of 3.20 and $2.99 \mathrm{eV}$, followed by the 1,3,5-linked polymer networks (3.05 and $2.87 \mathrm{eV}$ for F-CMP1 and S-CMP1, respectively): this is a result of the metalinkages in these polymers that limit the effective conjugation length. ${ }^{18}$ The 1,2,4,5-linked polymer networks are red-shifted compared to their 1,3,5-linked analogues, ${ }^{18}$ and they have similar optical gaps compared to their linear 1,4-benzene-linked analogues: $2.85 \mathrm{eV}$ for F-CMP2 versus $2.86 \mathrm{eV}$ for P3; $2.70 \mathrm{eV}$ for both S-CMP3 and P7. The $2,2^{\prime}, 7,7^{\prime}$-linked 9,9'-spirobifluorene networks ${ }^{18,49}$ and their linear 2,7-fluorene-linked analogues are the most red-shifted materials. Again, the polymer networks show similar optical gaps compared to their linear analogues, with optical gaps of $2.77 \mathrm{eV}$ for F-CMP3 and $2.75 \mathrm{eV}$ for P34. S-CMP3 has a gap of $2.56 \mathrm{eV}$ for F-CMP3 compared to $2.59 \mathrm{eV}$ for P35.

Prediction of difficult-to-measure (opto)electronic properties of polymer networks such as CMPs, for example, their ionization potential (IP) and electron affinity (EA), is challenging because of the complex structure of these materials. Therefore, we focused instead on predicting the relative properties of similarly sized cluster units of the different polymers. Otherwise, we used the same approach as in earlier work ${ }^{12,40,41,50}$ based around density functional theory (DFT) calculations with the B3LYP ${ }^{51,52}$ density functional and the $\mathrm{COSMO}^{53}$ dielectric screening model ( $\varepsilon_{\mathrm{r}} 80.1$, water). These calculations (Figure 3) predict that switching from fluorene to dibenzo[ $b, d]$ thiophene sulfone linker units results in the IP moving to more positive values, increasing the driving force for triethylamine (TEA) oxidation, in line with previous studies. ${ }^{12,16}$ However, these calculations also suggest that there should be no significant difference in potentials and driving
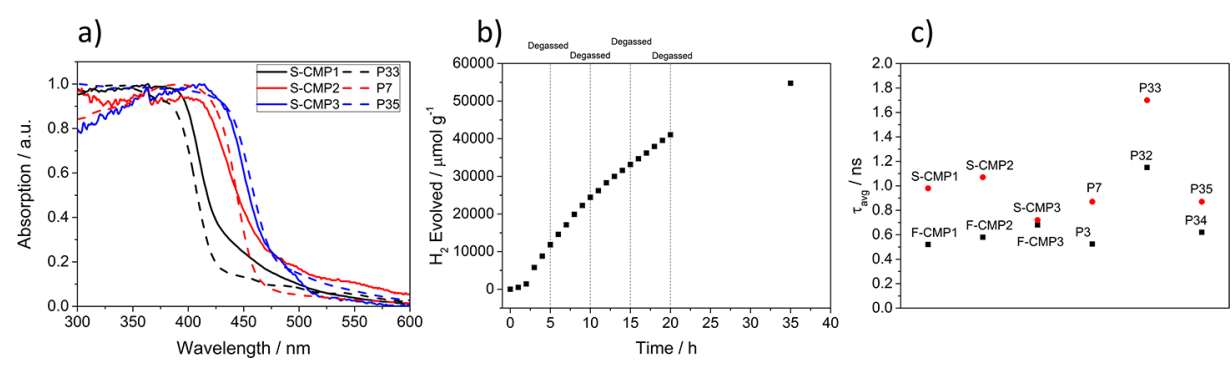

Figure 2. (a) UV/visible spectra of the six dibenzo[b,d]thiophene sulfone-containing photocatalysts as measured in the solid state; (b) plot showing sacrificial photocatalytic hydrogen evolution vs time for S-CMP3 under visible light irradiation $(\lambda>420 \mathrm{~nm}, 300 \mathrm{~W}$ Xe light source, $25 \mathrm{mg}$ of the photocatalyst in $25 \mathrm{~mL}$ of water/methanol/TEA mixture); (c) comparison of the weighted average fluorescence lifetimes measured for dibenzo $[b, d]$ thiophene sulfone and fluorene analogues. 


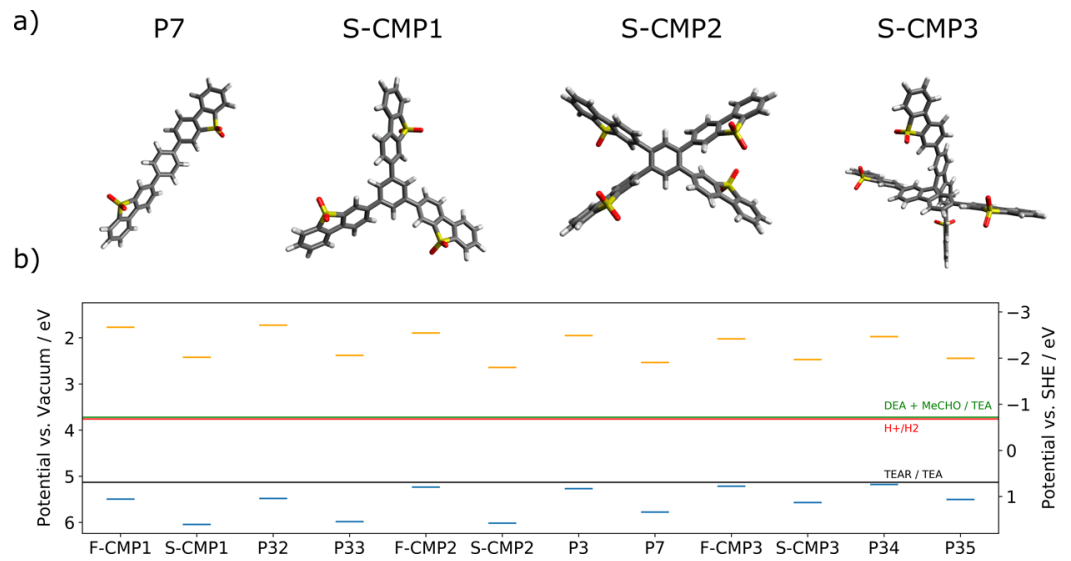

Figure 3. (a) B3LYP-/DZP-optimized structures of similar-sized cluster models of P7, S-CMP1, S-CMP2, and S-CMP3. (b) B3LYP-/DZPpredicted IP and EA potentials for similar-sized cluster models of the different polymers. Solution half reactions are shown as calculated for $\mathrm{pH} 11.5$ ( $\mathrm{MeCHO}$ acetaldehyde; TEAR-deprotonated TEA radical $\mathrm{N}(\mathrm{Et})_{2} \mathrm{CHCH}_{3}$ ). Potential for the oxidation of TEAR to DEA $+\mathrm{MeCHO}$ not shown as it is $<-2.5 \mathrm{~V}$. Underlying data tabulated in Tables S7 and S8. Note that because of the inherent small size of these cluster models, the absolute values of the potentials might deviate from those predicted here.

Table 1. Optical Properties, BET Surface Areas, and Photocatalytic HERs of the Polymer Photocatalysts, $\mathrm{TiO}_{2}$, and $\mathrm{C}_{3} \mathrm{~N}_{4}$

\begin{tabular}{|c|c|c|c|c|c|c|}
\hline material & optical gap ${ }^{a} / \mathrm{eV}$ & $\lambda_{\mathrm{em}}{ }^{b} / \mathrm{nm}$ & $\mathrm{SA}_{\mathrm{BET}}{ }^{c} / \mathrm{m}^{2} \mathrm{~g}^{-1}$ & HER $\lambda>420 \mathrm{~nm}^{d} / \mu \mathrm{mol} \mathrm{g}^{-1} \mathrm{~h}^{-1}$ & HER $\lambda>295 \mathrm{~nm}^{d} / \mu \mathrm{mol} \mathrm{g}^{-1} \mathrm{~h}^{-1}$ & $\tau_{\text {avg }}{ }^{e} / \mathrm{ns}$ \\
\hline F-CMP1 & 3.05 & 528 & 62 & 0.07 & $98.7( \pm 1.3)$ & 0.52 \\
\hline S-CMP1 & 2.87 & 450 & 508 & $667.0( \pm 22.0)$ & $1832.4( \pm 131.1)$ & 0.98 \\
\hline P32 & 3.20 & 420 & 20 & $<0.01$ & $230.1( \pm 10.5)$ & 1.15 \\
\hline P33 & 2.99 & 448 & 192 & $74.1( \pm 1.7)$ & $896.7( \pm 24.6)$ & 1.70 \\
\hline F-CMP2 & 2.85 & 439 & 51 & $<0.01$ & $8.0( \pm 3.8)$ & 0.58 \\
\hline S-CMP2 & 2.70 & 491 & 560 & $523.8( \pm 16.9)$ & $1482.7( \pm 51.9)$ & 1.07 \\
\hline P3 & 2.86 & 458,525 & 42 & $1.6( \pm 0.8)$ & $800.0( \pm 8.0)$ & 0.52 \\
\hline P7 & 2.70 & 477 & 69 & $1492.0( \pm 32)$ & $2352.1( \pm 76)$ & 0.87 \\
\hline F-CMP3 & 2.77 & 449 & 596 & $538.5( \pm 32.0)$ & $2545.3( \pm 21.5)$ & 0.68 \\
\hline S-CMP3 & 2.56 & 425,450 & 431 & $3106.0( \pm 202.4)$ & $6076.0( \pm 216.8)$ & 0.72 \\
\hline P34 & 2.75 & 462 & 11 & $47.5( \pm 3.2)$ & $128.7( \pm 1.0)$ & 0.62 \\
\hline P35 & 2.59 & 481 & 114 & $826.1( \pm 38.8)$ & $1176.8( \pm 75.9)$ & 0.87 \\
\hline $\mathrm{C}_{3} \mathrm{~N}_{4}^{f}$ & 2.70 & 460 & 22 & $108( \pm 4.0)$ & $448( \pm 24)$ & 4.24 \\
\hline $\mathrm{TiO}_{2}^{f}$ & 3.13 & 399 & 52 & 0 & $1492( \pm 52)$ & 0.65 \\
\hline
\end{tabular}

${ }^{a}$ Optical gap calculated from the absorption onset. ${ }^{b}$ Emission maximum $\left(\lambda_{\text {exc }}=360 \mathrm{~nm}\right) .{ }^{c}$ Apparent BET surface area calculated from the $\mathrm{N}_{2}$ adsorption isotherm. ${ }^{d}$ Reaction conditions: $25 \mathrm{mg}$ of the polymer was suspended in $25 \mathrm{~mL}$ of water/methanol/TEA solution, irradiated by a $300 \mathrm{~W}$ Xe lamp using suitable filters. ${ }^{e}$ Estimated weighted average lifetime of the excited state determined by time-correlated single-photon counting. Calculated by fitting the following equation: $A+B_{1} \times \exp \left(-i / \tau_{1}\right)+B_{2} \times \exp \left(-i / \tau_{2}\right)+B_{3} \times \exp \left(-i / \tau_{3}\right)$. Initial amplitudes $\left(A, B_{1}, B_{2}\right.$, and $\left.B_{3}\right)$ are estimated and iterated along with the lifetimes $\left(\tau_{1}, \tau_{2}\right.$, and $\left.\tau_{3}\right)$ until a fit is found. The prompt is measured separately and used for deconvolution of the instrument response. ${ }^{f}$ Loaded with 3 wt $\% \mathrm{Pt}$ from $\mathrm{H}_{2} \mathrm{PtCl}_{6}$.

force between the CMPs and the corresponding linear polymers. Finally, the torsion angles between adjacent units in the optimized polymer cluster models are $\sim 30^{\circ}$ larger for FCMP2 and S-CMP2 than for any of the other materials (see Table S9).

The photocatalytic activity of the polymers was studied under sacrificial conditions using both broad-spectrum irradiation $(\lambda>295 \mathrm{~nm})$ and visible light $(\lambda>420 \mathrm{~nm}$; Table 1). TEA was used as the sacrificial hole scavenger and methanol was added to avoid phase separation between water and the TEA. ${ }^{12,18}$ Methanol itself has been shown not to act as a hole scavenger for these photocatalysts. ${ }^{12}$ All photocatalysts were tested after purification by Soxhlet extraction, and without any additional metal cocatalysts (e.g., Pt), although significant residual levels of palladium $(0.13-1.61 \mathrm{wt} \%)$ were found by inductively coupled plasma optical emission spectrometry (see the Supporting Information), which has been shown previously to act as a cocatalyst for CMP photocatalysts, ${ }^{9,18}$ linear polymers, ${ }^{54} \mathrm{CTFs}^{29}$ and carbon nitride. $^{55}$

Palladium particles are distributed throughout both porous materials (F-CMP3 and S-CMP3) and the polar nonporous polymer (P35), as evident by transmission electron microscopy (TEM) measurements (Figures S15-S17). By using scanning mode and transmission mode images of the same area, we were also able to distinguish palladium particles on the surface from those within the material (Figures S18-S20). Palladium within CMPs has been previously reported to be accessible to aqueous solutions, ${ }^{56}$ and we also found that the palladium content of F-CMP3 and S-CMP3 could be reduced by washing with a palladium scavenger.

All of the materials in this series acted as photocatalysts for hydrogen evolution, albeit with large variations in activity (Table 1). As summarized in Figure 1, neither the porous CMPs nor the nonporous linear polymers can be said to be the "best" photocatalysts: for four pairings, the porous materials are superior (S-CMP1, F-CMP1, F-CMP3, and S-CMP3), 
whereas for two other pairings, the nonporous linear polymers give higher HERs (P3 and P7). The material with the highest overall HER, however, is microporous (S-CMP3). In the case of 1,3,5- and 1,3-linked polymers, F-CMP1 and P32 are virtually inactive under visible light $\left(0.07\right.$ and $<0.01 \mu \mathrm{mol} \mathrm{g}^{-1}$ $\mathrm{h}^{-1}$ ) and show low hydrogen production rates even under broadband illumination (98.7, $230.1 \mu \mathrm{mol} \mathrm{g} \mathrm{g}^{-1} \mathrm{~h}^{-1}$ ). These rates are similar to those observed previously for benzenelinked CMPs. ${ }^{18} \mathrm{~S}$-CMP1 has a rate of $667.0 \mu \mathrm{mol} \mathrm{g}^{-1} \mathrm{~h}^{-1}$ under visible light illumination and $1832.4 \mu \mathrm{mol} \mathrm{g}^{-1} \mathrm{~h}^{-1}$ under broadband illumination, strongly outperforming its linear 1,3benzene-linked analogue, P33 (74.1 and $896.7 \mu \mathrm{mol} \mathrm{g}^{-1} \mathrm{~h}^{-1}$, respectively). For the 1,4- and 1,2,4,5-linked polymers, the linear polymers, P3 $\left(1.6,800.0 \mu \mathrm{mol} \mathrm{g}^{-1} \mathrm{~h}^{-1}\right)$ and P7 (1492.0, 2352.1 $\left.\mu \mathrm{mol} \mathrm{g}^{-1} \mathrm{~h}^{-1}\right)$, have significantly higher HERs than their microporous analogues, F-CMP2 $\left(<0.01,8.0 \mu \mathrm{mol} \mathrm{g}^{-1}\right.$ $\left.\mathrm{h}^{-1}\right)$ and S-CMP2 $\left(523.8,1482.7 \mu \mathrm{mol} \mathrm{g}^{-1} \mathrm{~h}^{-1}\right)$. One potential reason might be that 1,2-substitutions in Suzuki-Miyaura reactions, which are needed to form the F-CMP2 and S-CMP2 materials, are challenging because of steric hindrance ${ }^{57}$ and thus defects may be expected, such as 1,3-linkages. This, taken together with the significantly larger torsion angles of the CMPs compared to their linear analogues, might limit the charge transport along the chain, as observed previously for conjugated materials. ${ }^{58,59}$ Related observations have been made by others in photocatalysis experiments where a 1,2,4,5-benzene-linked benzothiadiazole CMP was outperformed by the 1,3,5-benzene-linked analogue, which was itself outperformed by the corresponding 1,4-benzene-linked linear polymer. $^{22}$ For the fluorene-linked and 9,9'-spirobifluorenelinked polymers, this trend is reversed: the microporous polymers, F-CMP3 and S-CMP3, have much higher HERs than their nonporous linear analogues. For F-CMP3, the HER under broadband illumination is high $\left(2545.3 \mu \mathrm{mol} \mathrm{g} \mathrm{g}^{-1} \mathrm{~h}^{-1}\right)$, but because of its absorption onset at $447 \mathrm{~nm}$, it shows a lower hydrogen production rate under visible light $\left(538.5 \mu \mathrm{mol} \mathrm{g}^{-1}\right.$ $\mathrm{h}^{-1}$ ). S-CMP3 has the highest photocatalytic performance of the materials in this study under visible light irradiation: $3106.0 \mu \mathrm{mol} \mathrm{g}^{-1} \mathrm{~h}^{-1}$, which is much higher than its linear analogue P35 $\left(826.1 \mu \mathrm{mol} \mathrm{g} \mathrm{g}^{-1} \mathrm{~h}^{-1}\right)$. S-CMP3 shows a rate of $6076.0 \mu \mathrm{mol} \mathrm{g} \mathrm{g}^{-1}$ under broadband illumination, again higher than P35 under the same conditions $\left(1176.8 \mu \mathrm{mol} \mathrm{g}^{-1}\right.$ $\left.\mathrm{h}^{-1}\right)$. These HERs are among the highest reported for amorphous materials under sacrificial conditions.

In order to rule out the possibility that the difference in activity can be explained by the difference in residual $\mathrm{Pd}$ content, we synthesized F-CMP3 and S-CMP3 with varying amounts of the Pd catalyst. This resulted in materials that have different residual amounts of Pd (Tables S1 and S2), but similar optical onsets (Figures S31 and S32). For residual Pd amounts above 0.1 wt \%, we see little change in the photocatalytic activity of F-CMP3 and S-CMP3 (Figure S53). For the S-CMPs that contain the lowest amount of residual palladium $(470 \mathrm{ppm})$, a drop in performance to $1076.1 \mu \mathrm{mol} \mathrm{g}^{-1} \mathrm{~h}^{-1}$ is observed. We attempted to remove residual Pd from the samples with the lowest amount of Pd by washing the sample with a palladium scavenger. ${ }^{54,60}$ This reduced the amounts of residual palladium to $274 \mathrm{ppm}$ for FCMP3 and to $320 \mathrm{ppm}$ for S-CMP3 and greatly lowered their HERs, to 19.5 and $110.5 \mu \mathrm{mol} \mathrm{g}^{-1} \mathrm{~h}^{-1}$, respectively. The observation of an increase in activity followed by a plateau region has been reported previously for other $\mathrm{CMPs}^{9}$ and linear polymers. ${ }^{54}$ These results show that residual $\mathrm{Pd}$ is involved in the catalytic cycle but also that the differences in activity between the materials cannot be simply explained by differences in the amounts of residual Pd in the samples. When S-CMP3 containing $320 \mathrm{ppm}$ residual palladium was loaded with platinum via in situ photodeposition from $\mathrm{H}_{2} \mathrm{PtCl}_{6}$, an increase in catalytic activity was observed. A loading of $2.1 \mathrm{wt}$ $\%$ gave the highest HER, but the dependency on $\mathrm{Pt}$ concentration was not very large, with HERs varying between $1762 \mu \mathrm{mol} \mathrm{g}^{-1} \mathrm{~h}^{-1}$ (for $0.6 \mathrm{wt} \%$ Pt loading) and $2388 \mu \mathrm{mol}$ $\mathrm{g}^{-1} \mathrm{~h}^{-1}$ (for $2.1 \mathrm{wt} \% \mathrm{Pt}$ loading). At a higher loading of $7.1 \mathrm{wt}$ $\%$ platinum, the rate drops to $1124 \mu \mathrm{mol} \mathrm{g}^{-1} \mathrm{~h}^{-1}$. Similarly, a sample of S-CMP3 containing $8200 \mathrm{ppm}$ residual palladium showed a lower performance when loaded with additional 5.1 wt $\%$ platinum $\left(2210\right.$ vs $\left.250 \mu \mathrm{mol} \mathrm{g}^{-1} \mathrm{~h}^{-1}\right)$. This reduction in activity with higher metal loadings has been observed previously and it is related to hindered light absorption and enhanced recombination between photogenerated electrons and holes. ${ }^{55}$

An external quantum efficiency (EQE) of $7.7 \%( \pm 0.9 \%)$ was measured for platinized S-CMP3 at $420 \mathrm{~nm}$ in a water/ $\mathrm{MeOH} / \mathrm{TEA}$ mixture (the sample contains $320 \mathrm{ppm}$ residual palladium and $2.1 \mathrm{wt} \%$ platinum that was photodeposited onto the material). An even higher EQE of $13.2 \%( \pm 1.4 \%)$ was measured for as-synthesized S-CMP3 containing 0.72 wt \% palladium at $420 \mathrm{~nm}$ in a water/MeOH/TEA mixture. This value is significantly higher than previously reported EQEs for P1 $\left(\mathrm{EQE}_{420 \mathrm{~nm}}=0.4 \%\right)$, the linear dibenzo $[b, d]$ thiophene sulfone copolymer $\mathrm{P} 7\left(\mathrm{EQE}_{420 \mathrm{~nm}}=7.2 \%\right)$ under the same conditions. ${ }^{12}$ This efficiency also exceeds that reported for a platinum-modified tricyano-benzene-centered phenylenevinylene-co-terphenylene polymer network, OB-POP-3, in a triethanolamine mixture $\left(\mathrm{EQE}_{420 \mathrm{~nm}}=2.0 \%\right),{ }^{44}$ and $\mathrm{a}$ platinum-modified phenylene-benzothiadiazole-copolymer, B$\mathrm{BT}-1,4$, in a triethanolamine mixture $\left(\mathrm{EQE}_{420 \mathrm{~nm}}=4.0 \%\right){ }^{22}$ Wavelength-dependent photocatalytic experiments for $\mathrm{S}$ CMP1 (Figure S58) and S-CMP3 (Figure S59) show a decrease of activity with lowered absorptivity of both materials, roughly following their absorption profiles. This shows that the hydrogen production is a photocatalytic process, and it suggests that extension of the light absorption profile into visible and near infrared might further enhance the photocatalytic activity.

The photostability of S-CMP3 was evaluated by irradiating a sample for $35 \mathrm{~h}$ under $\lambda>420 \mathrm{~nm}$ illumination (Figure $2 \mathrm{~b}$ ). The hydrogen production rate was reduced over the course of the run, but the photocatalytic system remained active throughout. Furthermore, no apparent change in the FT-IR and UV/vis spectra was observed after the experiment (Figures S61 and S62).

The lifetime of the excited state was estimated using timecorrelated single-photon counting, with weighted averaged lifetimes ranging from 0.52 to $1.70 \mathrm{~ns}$. Unlike our previous study, ${ }^{18}$ we did not observe a clear correlation between the photocatalytic activity and the lifetime of the excited state for this series of materials (Figure S78). However, when comparing each subset of CMPs, the lifetime of the fluorene CMPs was always lower than the dibenzo[ $[b, d]$ thiophene sulfone CMP analogues (Figure 2c). For example, 1,3,5benzene-linked F-CMP1 has a lifetime of 0.52 ns compared to 0.98 ns for S-CMP1; likewise, the 1,2,4,5-benzene-linked FCMP2 has a shorter lifetime of 0.58 ns compared to S-CMP2 (1.07 ns). Only the lifetimes of 9,9'-spirobifluorene-linked FCMP3 and S-CMP3 are similar, and probably within the error 
of the measurement $(0.68$ and $0.72 \mathrm{~ns})$. We note here that the 9,9'-spirobifluorene S-CMP3 and F-CMP3 materials are also more similar in terms of their relative performance under broadband illumination and visible irradiation, as compared to the two other pairs of CMPs (S-CMP1 and F-CMP1 and SCMP2 and F-CMP2). The linear polymers show comparable trends: for example, the 1,4-benzene-linked dibenzo[b,d]thiophene sulfone copolymer P7 has a longer lifetime $(0.87$ ns) than the fluorene copolymer P3 (0.52 ns).

Particle size may also affect the photocatalytic activity, and we note that the powders for the fluorene materials in the solid state were in general coarser than for the dibenzo[b,d]thiophene sulfone analogues, though a previous study suggests that dispersibility, wettability, and interaction of water and scavenger with the dibenzo $[b, d]$ thiophene sulfone unit play a more important role. ${ }^{12}$ Scattering of light can affect the light harvesting significantly. This was studied by means of backscattering experiments (S82), and F-CMP3, S-CMP3, P7, P34, and P35 all show high backscattering values between 4 and $7 \%$.

Particle size distributions were measured by static lightscattering experiments in water/methanol/TEA mixtures, showing some variation. For each linear polymer/CMP pair, the Sauter mean diameter ${ }^{40}$ of the CMP networks was higher. Sauter mean diameters for the networks varied from 11.5 to 4.7 $\mu \mathrm{m}$, whereas the linear polymers showed ranged between 8.3 $\mu \mathrm{m}$ (P35) and $1.12 \mu \mathrm{m}$ (P7) (Table S5). This might indicate that the higher external surface area of smaller particles contributes to their catalytic activity: for instance, P7, easily the most active linear polymer, has by far the largest relative external surface area $\left(5365 \mathrm{~m}^{2} \mathrm{~cm}^{-3}\right)$, as compared to the structurally similar P33 $\left(1324 \mathrm{~m}^{2} \mathrm{~cm}^{-3}\right)$ and P35 $\left(725 \mathrm{~m}^{2}\right.$ $\left.\mathrm{cm}^{-3}\right)$, and also S-CMP3 $\left(523.8 \mathrm{~m}^{2} \mathrm{~cm}^{-3}\right)$. The observation that smaller particles have higher photocatalytic activity is also in line with reports on colloidal polymer photocatalysts. ${ }^{46,47,61}$

To investigate the possible effect of swelling, the particle sizes of P34, P35, F-CMP3, and S-CMP3 were also measured in water. The volume mean diameter of the two fluorene polymers decreased upon moving from water to water/TEA/ $\mathrm{MeOH}$, whereas the dibenzo $[b, d]$ thiophene sulfone analogues increased in size when moving from water to water/TEA/ $\mathrm{MeOH}$. This size increase for the network S-CMP3 was significantly larger (factor of 4.4) than for the analogous linear polymer P35 (factor of 1.67).

This may indicate that S-CMP3 swells in the reaction mixture, thus further increasing the catalytically active surface area in comparison with the other materials. This perhaps shows that to gain a comprehensive insight into the catalytically active surface area in the reaction mixture, other factors, such as swellability and water uptake, need to be considered in addition to dry-state measurements by gas adsorption.

The photocatalytic performance within the polymer series could not be correlated simply with BET surface area measurements (Figure S73). However, water uptake measurements for F-CMP3 and S-CMP3 show distinct differences: FCMP3 shows very little water uptake in the relative pressure region of $0.2-0.6 P / P_{0}$, but uptake is observed in this region for the dibenzo $[b, d]$ thiophene sulfone material S-CMP3 (Figure 4). This means that water can penetrate the pore structure in the more polar S-CMP3 to a greater extent than it does in F-CMP3, which might contribute to the observed difference in photocatalytic activity. At higher relative

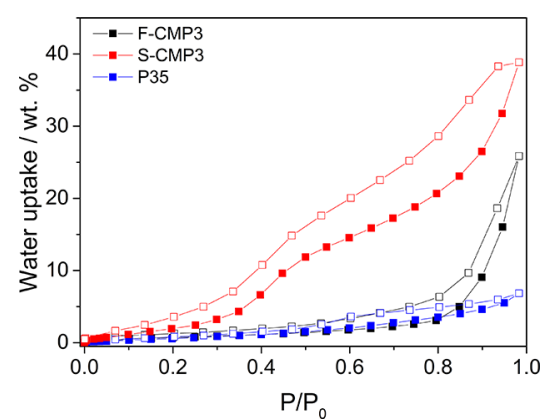

Figure 4. Water uptake measurements at $20.0{ }^{\circ} \mathrm{C}$. Solid squares: adsorption, open squares: desorption.

pressures, both materials exhibit water uptake, and this relates to free water that is adsorbed on the surface and interstitial space of the particles. ${ }^{62}$

Very little water uptake was observed for P35, the linear analogue of S-CMP3, which again could contribute to the higher performance of S-CMP3 compared to its nonporous analogue, P35. As discussed above, we found that swellability is evident in S-CMP3 in water/methanol/TEA mixtures. Furthermore, organic molecules such as TEA and methanol affect the wetting of these materials. When $\mathrm{Na}_{2} \mathrm{~S}$ was used instead as the sacrificial scavenger, a reduction in activity under visible light was observed to $10.3 \mu \mathrm{mol} \mathrm{g}^{-1} \mathrm{~h}^{-1}$ for F-CMP3, to $216 \mu \mathrm{mol} \mathrm{g}^{-1} \mathrm{~h}^{-1}$ for S-CMP3, and to $68 \mu \mathrm{mol} \mathrm{g}^{-1} \mathrm{~h}^{-1}$ for P35. Compared to the experiments in water/methanol/TEA, the reduction is largest for F-CMP3 (52 times) compared to SCMP3 (14 times) and P35 (12 times). Similarly, photocurrent measurements (Figure S60) in a sodium sulfate solution showed that the response for porous and wettable S-CMP3 was greater than its nonporous analogue, P35, as well as its porous, nonpolar analogue, F-CMP3. Taken together, these data suggest that a combination of high surface area and wettability seems to be beneficial for aqueous photocatalysis in water, providing that the provision of these surface properties does not negatively impact other factors, such as the potentials of the charge carriers (IP, EA) that drive the oxidation of TEA and reduction of protons. The latter is not the case here, as predicted by DFT calculations (Figure 3 ).

To study the differences between F-CMP3 and S-CMP3 further, we performed, for the first time, large-scale, facilitybased neutron-scattering measurements on CMPs. Incoherent quasi-elastic neutron scattering (QENS) allowed us to study the difference in dynamics between dry and hydrated CMPs, as well as to probe trapped water in F-CMP3 and S-CMP3 pore networks, on a 5-50 ps time scale. The large difference in incoherent neutron cross sections between carbons and hydrogens means that the QENS signal is largely dominated by the water signal. However, the use of deuterated water $\left(\mathrm{D}_{2} \mathrm{O}\right)$ allows us to reduce the water signal significantly due to the much lower incoherent neutron cross sections of deuterium.

First, we studied the CMPs in $\mathrm{D}_{2} \mathrm{O}$ to extract the QENS signal of the hydrated CMPs. The $Q$-averaged dynamical structure factor $S(E)$ at $300 \mathrm{~K}$ is plotted in Figure S83. Figure S83a,b show the impact of hydrating F-CMP3 and S-CMP3, respectively. In both cases, the QENS signal broadens as the amount of $\mathrm{D}_{2} \mathrm{O}$ increases and a background develops for a $\mathrm{D}_{2} \mathrm{O}$ content higher than $32 \mathrm{wt} \%$ for F-CMP3 and $52 \mathrm{wt} \%$ for $S-C M P 3$, respectively. The QENS signal at higher $\mathrm{D}_{2} \mathrm{O}$ content for both samples can be fitted reasonably by 
a)

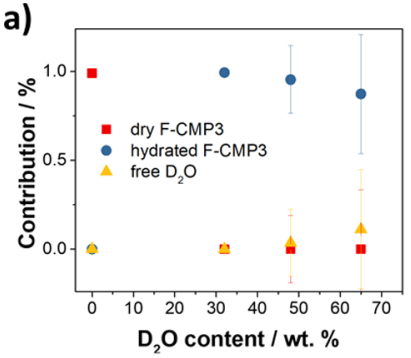

d)

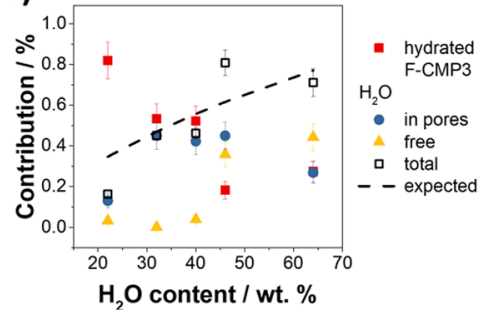

b)

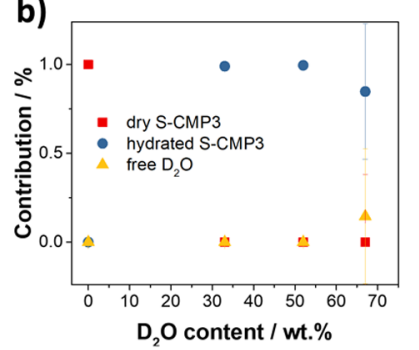

e)

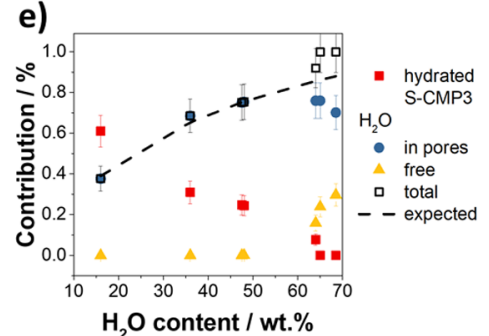

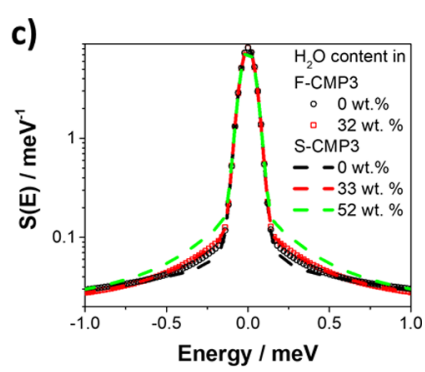

f)

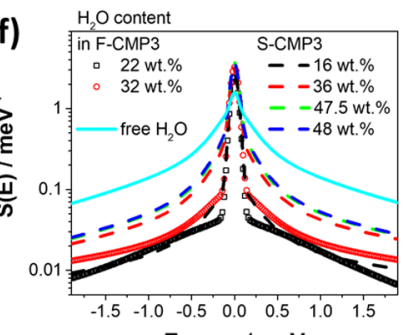

Figure 5. Dry, hydrated CMP and free $\mathrm{D}_{2} \mathrm{O}$ contributions to the QENS (incoherent QENS) signals of Figure S83a,b for (a) F-CMP3 and (b) SCMP3. (c) QENS fit of dry and hydrated F-CMP3 and S-CMP3. Hydrated CMP and trapped and free water contributions to the QENS signals of Figure S83c,d for F-CMP3 (d) and S-CMP3 (e). The dotted lines represent the expected $\mathrm{H}_{2} \mathrm{O}$ contribution based on the mass ratio and difference in the incoherent neutron cross sections between CMP and $\mathrm{H}_{2} \mathrm{O}$. (f) QENS fit of the trapped water contribution.

combining the signal of pure $\mathrm{D}_{2} \mathrm{O}$ and F-CMP3: $\mathrm{D}_{2} \mathrm{O} 32$ wt \%, and S-CMP3: $\mathrm{D}_{2} \mathrm{O} 52$ wt \%, respectively (Figures S84 and S86). Thus, we assign this background to free water and this onset to a maximum water uptake (Figure 5a,b). Comparing hydrated F-CMP3 and S-CMP3 QENS signals (Figure 5c) points toward faster dynamics of S-CMP3 on the time scale studied. To summarize, S-CMP3 exhibits faster dynamics on 5-50 ps time scale in the fully hydrated states than F-CMP3 and it absorbs more water (52 wt \%) than F-CMP3 (32 wt \%). These water uptake values calculated from QENS correlate well with the water uptake measurements (Figure 4).

Next, we investigated the water dynamics within the pores of F-CMP3 and S-CMP3. Figure S83c,d shows the impact of the water content on the dynamics of some specific states of water. At high water content, the QENS spectra resemble the free water signal but at a low water content, a clear elastic contribution arising from the CMP itself can be observed.

It was not possible to fit the QENS signals by using a combination of the hydrated CMP signal (extracted from the previous measurements with $\mathrm{D}_{2} \mathrm{O}$ ) and the pure water signal. We therefore fitted these QENS data by adding a third contribution (modeled by a delta function and two Lorentzians convoluted by the resolution of the instrument) that we assign to the dynamics of water in the pores. Below $32 \mathrm{wt} \% \mathrm{H}_{2} \mathrm{O}$ for F-CMP3 and 52 wt $\% \mathrm{H}_{2} \mathrm{O}$ for S-CMP3, respectively, the QENS signal can be modeled by a combination of the hydrated CMP signal and the signal from the water in the pores, as shown in Figures $\mathrm{S} 85$ and S87. For higher $\mathrm{H}_{2} \mathrm{O}$ contents, the QENS signal is modeled reasonably by a combination of the hydrated CMP signal, the free $\mathrm{H}_{2} \mathrm{O}$ signal, and the signal from the water in the pores. The fraction of all the contributions is shown in Figure 5d,e. The free $\mathrm{H}_{2} \mathrm{O}$ contribution lies below the expected water contribution, as calculated using the mass ratio and the difference in the incoherent cross section between the CMP molecules and $\mathrm{H}_{2} \mathrm{O}$. By adding the third contribution to the free $\mathrm{H}_{2} \mathrm{O}$ contribution, we obtain a total $\mathrm{H}_{2} \mathrm{O}$ contribution (red squares in Figure $5 \mathrm{~d}, \mathrm{e})$ that is in reasonable agreement with the expected $\mathrm{H}_{2} \mathrm{O}$ contribution, confirming our assignment of the extra contribution to the dynamics of water in the pore networks of the CMPs. Figure $5 \mathrm{f}$ shows that there is an increase in the elastic contribution and a narrowing of the QENS signal for the water in the pores in comparison with free water. This indicates that the dynamics of the water in the pores is frustrated on the accessible $5-50$ ps time scale. This effect is more pronounced in the case of F-CMP3.

To our knowledge, this is the first time that QENS has been applied to probe local dynamics of CMPs. In the accessible 550 ps instrumental time scale, we have shown that the dynamics of the F-CMP3 and S-CMP3 in a dry state are similar, but the dynamics of S-CMP3 in a fully hydrated state are faster than for F-CMP3. This correlates with S-CMP3 adsorbing more water than F-CMP3. We also show that the water dynamics is frustrated up to the maximum water uptake due to pore confinement; this frustration effect is more pronounced in the case of F-CMP3. The lower water uptake of F-CMP3 in comparison to S-CMP3, combined with more frustrated water dynamics, points toward reduced mass transport in the F-CMP3 network that may contribute to its reduced photocatalytic activity.

\section{CONCLUSIONS}

The introduction of 9,9'-spirobifluorene linkers in S-CMP3 results in a microporous material with a HER that is more than three times higher than the linear nonporous analogue, P35, under visible irradiation, and more than five times higher under broadband illumination. These rates are among the highest observed for organic polymers. By contrast, porous analogues of copolymers P3 and P7 show reduced photocatalytic activities. In both cases, the observed optical gaps and light absorption profiles for the porous and nonporous analogues are relatively similar (Figure $2 \mathrm{a}$ ). This seems to originate in the highly twisted nature of the CMPs because of the linker used, which may cause a reduction in charge mobility in the lowdensity porous materials, which might in turn counterbalance or even negate any mass-transfer benefits that arise from 
porosity. As observed previously, dibenzo $[b, d]$ thiophene sulfone-containing materials show significantly enhanced HERs with respect to fluorene analogues, and this is true across the whole series of polymers. This study suggests that CMPs may yet have advantages as components for overall water splitting schemes, and it is a CMP, S-CMP3, that shows the highest performance in this study. Although no global correlation between the excited state lifetime and the HER was found, it seems that the dibenzo[ $[b, d]$ thiophene sulfone units prolong exciton separation, and this may contribute toward increased activity over fluorene analogues, alongside other factors such as red-shifted absorption onsets and enhanced wettability of the material particles and its pores. We have also demonstrated the relevance of QENS measurements for studying the structure-property relationship of CMPs in the context of photocatalysis. A forthcoming work will be dedicated to a detailed study of the Q-dependence of the QENS spectra. This is expected to provide deeper insights into the mass transport processes in these CMP networks.

\section{ASSOCIATED CONTENT}

\section{S Supporting Information}

The Supporting Information is available free of charge on the ACS Publications website at DOI: 10.1021/acs.chemmater. 8 b02833.

Full synthetic details, additional synthetic experiments and analysis for all materials, TGA, FT-IR, PXRD, gas sorption, UV/vis and photoluminescence spectra, SEM, TEM, energy-dispersive X-ray data, computational data, further photocatalysis data, time-correlated singlephoton counting data, selective laser sintering, and QENS data (PDF)

\section{AUTHOR INFORMATION}

\section{Corresponding Authors}

*E-mail: ssprick@liverpool.ac.uk (R.S.S.).

*E-mail: aicooper@liverpool.ac.uk (A.I.C.).

ORCID $\odot$

Reiner Sebastian Sprick: 0000-0002-5389-2706

Yang Bai: 0000-0002-1643-3770

Anne A. Y. Guilbert: 0000-0003-3369-7900

Catherine M. Aitchison: 0000-0003-1437-8314

Duncan J. Woods: 0000-0003-0595-5941

Martijn A. Zwijnenburg: 0000-0001-5291-2130

Andrew I. Cooper: 0000-0003-0201-1021

\section{Author Contributions}

This manuscript was written through contributions from all authors.

\section{Notes}

The authors declare no competing financial interest.

\section{ACKNOWLEDGMENTS}

We thank the Engineering and Physical Sciences Research Council (EPSRC) for the financial support under Grant EP/ N004884/1. Y.B. thanks the China Scholarship Council for a Ph.D. studentship. A.A.Y.G. thanks EPSRC for an EPSRC Postdoctoral Fellowship (EP/P00928X/1). C.M.A. thanks the EPSRC for financial support through a DTP grant. The Institut Laue-Langevin (ILL) facility (Grenoble, France) is acknowledged for providing beam time on the IN6 spectrometer. The Imaging Centre at Liverpool (ICaL) is acknowledged for performing TEM imaging. R.S.S. thanks Dr. T. Hasell for helpful discussions.

\section{REFERENCES}

(1) Kudo, A.; Miseki, Y. Heterogeneous Photocatalyst Materials for Water Splitting. Chem. Soc. Rev. 2009, 38, 253-278.

(2) Martin, D. J.; Reardon, P. J. T.; Moniz, S. J. A.; Tang, J. Visible Light-Driven Pure Water Splitting by a Nature-Inspired Organic Semiconductor-Based System. J. Am. Chem. Soc. 2014, 136, 1256812571.

(3) Wang, Q.; Hisatomi, T.; Suzuki, Y.; Pan, Z.; Seo, J.; Katayama, M.; Minegishi, T.; Nishiyama, H.; Takata, T.; Seki, K.; et al. Particulate Photocatalyst Sheets Based on Carbon Conductor Layer for Efficient Z-Scheme Pure-Water Splitting at Ambient Pressure. J. Am. Chem. Soc. 2017, 139, 1675-1683.

(4) Wang, Q.; Hisatomi, T.; Jia, Q.; Tokudome, H.; Zhong, M.; Wang, C.; Pan, Z.; Takata, T.; Nakabayashi, M.; Shibata, N.; et al. Scalable Water Splitting on Particulate Photocatalyst Sheets with a Solar-to-Hydrogen Energy Conversion Efficiency Exceeding 1\%. Nat. Mater. 2016, 15, 611-615.

(5) Wang, X.; Maeda, K.; Thomas, A.; Takanabe, K.; Xin, G.; Carlsson, J. M.; Domen, K.; Antonietti, M. A Metal-Free Polymeric Photocatalyst for Hydrogen Production from Water under Visible Light. Nat. Mater. 2008, 8, 76-80.

(6) Ong, W.-J.; Tan, L.-L.; Ng, Y. H.; Yong, S.-T.; Chai, S.-P. Graphitic Carbon Nitride (g-C3N4)-Based Photocatalysts for Artificial Photosynthesis and Environmental Remediation: Are We a Step Closer To Achieving Sustainability? Chem. Rev. 2016, 116, 7159-7329.

(7) Schwinghammer, K.; Mesch, M. B.; Duppel, V.; Ziegler, C.; Senker, J.; Lotsch, B. V. Crystalline Carbon Nitride Nanosheets for Improved Visible-Light Hydrogen Evolution. J. Am. Chem. Soc. 2014, $136,1730-1733$.

(8) Zhang, G.; Li, G.; Lan, Z.-A.; Lin, L.; Savateev, A.; Heil, T.; Zafeiratos, S.; Wang, X.; Antonietti, M. Optimizing Optical Absorption, Exciton Dissociation, and Charge Transfer of a Polymeric Carbon Nitride with Ultrahigh Solar Hydrogen Production Activity. Angew. Chem., Int. Ed. 2017, 56, 13445-13449.

(9) Li, L.; Cai, Z.; Wu, Q.; Lo, W.-Y.; Zhang, N.; Chen, L. X.; Yu, L. Rational Design of Porous Conjugated Polymers and Roles of Residual Palladium for Photocatalytic Hydrogen Production. J. Am. Chem. Soc. 2016, 138, 7681-7686.

(10) Woods, D. J.; Sprick, R. S.; Smith, C. L.; Cowan, A. J.; Cooper, A. I. A Solution-Processable Polymer Photocatalyst for Hydrogen Evolution from Water. Adv. Energy Mater. 2017, 7, 1700479.

(11) Zong, X.; Miao, X.; Hua, S.; An, L.; Gao, X.; Jiang, W.; Qu, D.; Zhou, Z.; Liu, X.; Sun, Z. Structure Defects Assisted Photocatalytic H2 Production for Polythiophene Nanofibers. Appl. Catal. B Environ. 2017, 211, 98-105.

(12) Sprick, R. S.; Bonillo, B.; Clowes, R.; Guiglion, P.; Brownbill, N. J.; Slater, B. J.; Blanc, F.; Zwijnenburg, M. A.; Adams, D. J.; Cooper, A. I. Visible-Light-Driven Hydrogen Evolution Using Planarized Conjugated Polymer Photocatalysts. Angew. Chem., Int. Ed. 2015, 55, 1792-1796.

(13) Yanagida, S.; Kabumoto, A.; Mizumoto, K.; Pac, C.; Yoshino, K. Poly(p-phenylene)-Catalysed Photoreduction of Water to Hydrogen. J. Chem. Soc., Chem. Commun. 1985, 474-475.

(14) Yanagida, S.; Ogata, T.; Kuwana, Y.; Wada, Y.; Murakoshi, K.; Ishida, A.; Takamuku, S.; Kusaba, M.; Nakashima, N. Synthesis of $2,^{\prime}: 5^{\prime}, 2^{\prime \prime}$-terpyridine and $2,2^{\prime}: 5^{\prime}, 2^{\prime \prime}: 5^{\prime \prime}, 2^{\prime \prime \prime}$-quaterpyridine and their photocatalysis of the reduction of water. J. Chem. Soc. Perkin Trans. 2 1996, 1963-1969.

(15) Dai, C.; Xu, S.; Liu, W.; Gong, X.; Panahandeh-Fard, M.; Liu, Z.; Zhang, D.; Xue, C.; Loh, K. P.; Liu, B. Dibenzothiophene-S ,S -Dioxide-Based Conjugated Polymers: Highly Efficient Photocatalyts for Hydrogen Production from Water under Visible Light. Small 2018, 14, 1801839.

(16) Sachs, M.; Sprick, R. S.; Pearce, D.; Hillman, S. A. J.; Monti, A.; Guilbert, A. A. Y.; Brownbill, N. J.; Dimitrov, S.; Shi, X.; Blanc, F.; 
et al. Understanding Structure-Activity Relationships in Linear Polymer Photocatalysts for Hydrogen Evolution. Nat. Commun. 2018, 9, 4968.

(17) Sprick, R. S.; Jiang, J.-X.; Bonillo, B.; Ren, S.; Ratvijitvech, T.; Guiglion, P.; Zwijnenburg, M. A.; Adams, D. J.; Cooper, A. I. Tunable Organic Photocatalysts for Visible-Light-Driven Hydrogen Evolution. J. Am. Chem. Soc. 2015, 137, 3265-3270.

(18) Sprick, R. S.; Bonillo, B.; Sachs, M.; Clowes, R.; Durrant, J. R.; Adams, D. J.; Cooper, A. I. Extended Conjugated Microporous Polymers for Photocatalytic Hydrogen Evolution from Water. Chem. Commun. 2016, 52, 10008-10011.

(19) Li, L.; Lo, W.-y.; Cai, Z.; Zhang, N.; Yu, L. Donor-Acceptor Porous Conjugated Polymers for Photocatalytic Hydrogen Production: The Importance of Acceptor Comonomer. Macromolecules 2016, 49, 6903-6909.

(20) Wang, L.; Wan, Y.; Ding, Y.; Niu, Y.; Xiong, Y.; Wu, X.; Xu, H. Photocatalytic Oxygen Evolution from Low-Bandgap Conjugated Microporous Polymer Nanosheets: A Combined First-Principles Calculation and Experimental Study. Nanoscale 2017, 9, 4090-4096.

(21) Li, L.; Cai, Z. Structure Control and Photocatalytic Performance of Porous Conjugated Polymers Based on Perylene Diimide. Polym. Chem. 2016, 7, 4937-4943.

(22) Yang, C.; Ma, B. C.; Zhang, L.; Lin, S.; Ghasimi, S.; Landfester, K.; Zhang, K. A. I.; Wang, X. Molecular Engineering of Conjugated Polybenzothiadiazoles for Enhanced Hydrogen Production by Photosynthesis. Angew. Chem., Int. Ed. 2016, 55, 9202-9206.

(23) Xiang, Y.; Wang, X.; Rao, L.; Wang, P.; Huang, D.; Ding, X.; Zhang, X.; Wang, S.; Chen, H.; Zhu, Y. Conjugated Polymers with Sequential Fluorination for Enhanced Photocatalytic H 2 Evolution via Proton-Coupled Electron Transfer. ACS Energy Lett 2018, 3, 2544.

(24) Cheng, C.; Wang, X.; Lin, Y.; He, L.; Jiang, J.-X.; Xu, Y.; Wang, F. The Effect of Molecular Structure and Fluorination on the Properties of Pyrene-Benzothiadiazole-Based Conjugated Polymers for Visible-Light-Driven Hydrogen Evolution. Polym. Chem. 2018, 9, $4468-4475$.

(25) Wang, Z.; Yang, X.; Yang, T.; Zhao, Y.; Wang, F.; Chen, Y.; Zeng, J. H.; Yan, C.; Huang, F.; Jiang, J.-X. Dibenzothiophene Dioxide Based Conjugated Microporous Polymers for Visible-Light-Driven Hydrogen Production. ACS Catal. 2018, 8, 8590-8596.

(26) Bi, J.; Fang, W.; Li, L.; Wang, J.; Liang, S.; He, Y.; Liu, M.; Wu, L. Covalent Triazine-Based Frameworks as Visible Light Photocatalysts for the Splitting of Water. Macromol. Rapid Commun. 2015, 36, 1799-1805.

(27) Schwinghammer, K.; Hug, S.; Mesch, M. B.; Senker, J.; Lotsch, B. V. Phenyl-Triazine Oligomers for Light-Driven Hydrogen Evolution. Energy Environ. Sci. 2015, 8, 3345-3353.

(28) Kuecken, S.; Acharjya, A.; Zhi, L.; Schwarze, M.; Schomäcker, R.; Thomas, A. Fast Tuning of Covalent Triazine Frameworks for Photocatalytic Hydrogen Evolution. Chem. Commun. 2017, 53, 58545857.

(29) Meier, C. B.; Sprick, R. S.; Monti, A.; Guiglion, P.; Lee, J.-S. M.; Zwijnenburg, M. A.; Cooper, A. I. Structure-Property Relationships for Covalent Triazine-Based Frameworks: The Effect of Spacer Length on Photocatalytic Hydrogen Evolution from Water. Polymer 2017, 126, 283-290.

(30) Zhang, Z.; Long, J.; Yang, L.; Chen, W.; Dai, W.; Fu, X.; Wang, $\mathrm{X}$. Organic semiconductor for artificial photosynthesis: water splitting into hydrogen by a bioinspired C3N3S3polymer under visible light irradiation. Chem. Sci. 2011, 2, 1826-1830.

(31) Chu, S.; Wang, Y.; Guo, Y.; Zhou, P.; Yu, H.; Luo, L.; Kong, F.; Zou, Z. Facile Green Synthesis of Crystalline Polyimide Photocatalyst for Hydrogen Generation from Water. J. Mater. Chem. 2012, 22, 15519-15521.

(32) Lan, Z.-A.; Fang, Y.; Zhang, Y.; Wang, X. Photocatalytic Oxygen Evolution from Functional Triazine-Based Polymers with Tunable Band Structures. Angew. Chem., Int. Ed. 2017, 57, 470-474.

(33) Guo, L.; Niu, Y.; Xu, H.; Li, Q.; Razzaque, S.; Huang, Q.; Jin, S.; Tan, B. Engineering heteroatoms with atomic precision in donor- acceptor covalent triazine frameworks to boost photocatalytic hydrogen production. J. Mater. Chem. A 2018, 6, 19775-19781.

(34) Schwarz, D.; Acharjya, A.; Ichangi, A.; Kochergin, Y. S.; Lyu, P.; Opanasenko, M. V.; Tarábek, J.; Vacek Chocholoušová, J.; Vacek, J.; Schmidt, J.; et al. Tuning the Porosity and Photocatalytic Performance of Triazine-Based Graphdiyne Polymers through Polymorphism. ChemSusChem 2018, DOI: 10.1002/cssc.201802034.

(35) Vyas, V. S.; Haase, F.; Stegbauer, L.; Savasci, G.; Podjaski, F.; Ochsenfeld, C.; Lotsch, B. V. A Tunable Azine Covalent Organic Framework Platform for Visible Light-Induced Hydrogen Generation. Nat. Commun. 2015, 6, 8508.

(36) Haase, F.; Banerjee, T.; Savasci, G.; Ochsenfeld, C.; Lotsch, B. V. Structure-property-activity Relationships in a Pyridine Containing Azine-Linked Covalent Organic Framework for Photocatalytic Hydrogen Evolution. Faraday Discuss. 2017, 201, 247-264.

(37) Stegbauer, L.; Schwinghammer, K.; Lotsch, B. V. A HydrazoneBased Covalent Organic Framework for Photocatalytic Hydrogen Production. Chem. Sci. 2014, 5, 2789-2793.

(38) Wang, X.; Chen, L.; Chong, S. Y.; Little, M. A.; Wu, Y.; Zhu, W.-H.; Clowes, R.; Yan, Y.; Zwijnenburg, M. A.; Sprick, R. S.; et al. Sulfone-Containing Covalent Organic Frameworks for Photocatalytic Hydrogen Evolution from Water. Nat. Chem. 2018, 10, 1180-1189.

(39) Pachfule, P.; Acharjya, A.; Roeser, J.; Langenhahn, T.; Schwarze, M.; Schomäcker, R.; Thomas, A.; Schmidt, J. Diacetylene Functionalized Covalent Organic Framework (COF) for Photocatalytic Hydrogen Generation. J. Am. Chem. Soc. 2018, 140, 14231427.

(40) Sprick, R. S.; Aitchison, C. M.; Berardo, E.; Turcani, L.; Wilbraham, L.; Alston, B. M.; Jelfs, K. E.; Zwijnenburg, M. A.; Cooper, A. I. Maximising the Hydrogen Evolution Activity in Organic Photocatalysts by Co-Polymerisation. J. Mater. Chem. A 2018, 6, 11994-12003.

(41) Sprick, R. S.; Wilbraham, L.; Bai, Y.; Guiglion, P.; Monti, A.; Clowes, R.; Cooper, A. I.; Zwijnenburg, M. A. Nitrogen Containing Linear Poly(phenylene) Derivatives for Photo-Catalytic Hydrogen Evolution from Water. Chem. Mater. 2018, 30, 5733-5742.

(42) Xu, Y.; Mao, N.; Zhang, C.; Wang, X.; Zeng, J.; Chen, Y.; Wang, F.; Jiang, J.-X. Rational design of donor- $\pi$-acceptor conjugated microporous polymers for photocatalytic hydrogen production. Appl. Catal. B Environ. 2018, 228, 1-9.

(43) Kochergin, Y. S.; Schwarz, D.; Acharjya, A.; Ichangi, A.; Kulkarni, R.; Eliášová, P.; Vacek, J.; Schmidt, J.; Thomas, A.; Bojdys, M. J. Exploring the "Goldilocks Zone" of Semiconducting Polymer Photocatalysts by Donor-Acceptor Interactions. Angew. Chem., Int. Ed. 2018, 57, 14188-14192.

(44) Bi, S.; Lan, Z.-A.; Paasch, S.; Zhang, W.; He, Y.; Zhang, C.; Liu, F.; Wu, D.; Zhuang, X.; Brunner, E.; et al. Substantial CyanoSubstituted Fully sp2 -Carbon-Linked Framework: Metal-Free Approach and Visible-Light-Driven Hydrogen Evolution. Adv. Funct. Mater. 2017, 27, 1703146

(45) Xu, Y.; Mao, N.; Feng, S.; Zhang, C.; Wang, F.; Chen, Y.; Zeng, J.; Jiang, J.-X. Perylene-Containing Conjugated Microporous Polymers for Photocatalytic Hydrogen Evolution. Macromol. Chem. Phys. 2017, 218, 1700049 .

(46) Wang, L.; Fernández-Terán, R.; Zhang, L.; Fernandes, D. L. A.; Tian, L.; Chen, H.; Tian, H. Organic Polymer Dots as Photocatalysts for Visible Light-Driven Hydrogen Generation. Angew. Chem., Int. Ed. 2016, 55, 12306-12310.

(47) Tseng, P.-J.; Chang, C.-L.; Chan, Y.-H.; Ting, L.-Y.; Chen, P.Y.; Liao, C.-H.; Tsai, M.-L.; Chou, H.-H. Design and Synthesis of Cycloplatinated Polymer Dots as Photocatalysts for Visible-LightDriven Hydrogen Evolution. ACS Catal. 2018, 8, 7766-7772.

(48) Pati, P. B.; Damas, G.; Tian, L.; Fernandes, D. L. A.; Zhang, L.; Pehlivan, I. B.; Edvinsson, T.; Araujo, C. M.; Tian, H. An Experimental and Theoretical Study of an Efficient Polymer NanoPhotocatalyst for Hydrogen Evolution. Energy Environ. Sci. 2017, 10, $1372-1376$

(49) Weber, J.; Thomas, A. Toward Stable Interfaces in Conjugated Polymers: Microporous Poly(p-Phenylene) and Poly- 
(Phenyleneethynylene) Based on a Spirobifluorene Building Block. J. Am. Chem. Soc. 2008, 130, 6334-6335.

(50) Guiglion, P.; Butchosa, C.; Zwijnenburg, M. A. Polymeric Watersplitting Photocatalysts; a Computational Perspective on the Water Oxidation Conundrum. J. Mater. Chem. A 2014, 2, 1199612004.

(51) Becke, A. D. Density-functional Thermochemistry. III. The Role of Exact Exchange. J. Chem. Phys. 1993, 98, 5648-5652.

(52) Stephens, P. J.; Devlin, F. J.; Chabalowski, C. F.; Frisch, M. J. $\mathrm{Ab}$ Initio Calculation of Vibrational Absorption and Circular Dichroism Spectra Using Density Functional Force Fields. J. Phys. Chem. 1994, 98, 11623-11627.

(53) Klamt, A.; Schüürmann, G. COSMO: a new approach to dielectric screening in solvents with explicit expressions for the screening energy and its gradient. J. Chem. Soc., Perkin Trans. 2 1993, 799-805.

(54) Kosco, J.; Sachs, M.; Godin, R.; Kirkus, M.; Francas, L.; Bidwell, M.; Qureshi, M.; Anjum, D.; Durrant, J. R.; McCulloch, I. The Effect of Residual Palladium Catalyst Contamination on the Photocatalytic Hydrogen Evolution Activity of Conjugated Polymers. Adv. Energy Mater. 2018, 8, 1802181.

(55) Maeda, K.; Wang, X.; Nishihara, Y.; Lu, D.; Antonietti, M.; Domen, K. Photocatalytic Activities of Graphitic Carbon Nitride Powder for Water Reduction and Oxidation under Visible Light. J. Phys. Chem. C 2009, 113, 4940-4947.

(56) Wang, F.; Mielby, J.; Richter, F. H.; Wang, G.; Prieto, G.; Kasama, T.; Weidenthaler, C.; Bongard, H.-J.; Kegnaes, S.; Fürstner, A.; et al. A Polyphenylene Support for Pd Catalysts with Exceptional Catalytic Activity. Angew. Chem., Int. Ed. 2014, 53, 8645-8648.

(57) Yin, J.; Rainka, M. P.; Zhang, X.-X.; Buchwald, S. L. A Highly Active Suzuki Catalyst for the Synthesis of Sterically Hindered Biaryls: Novel Ligand Coordination. J. Am. Chem. Soc. 2002, 124, 1162-1163.

(58) Yu, Z. G.; Smith, D. L.; Saxena, A.; Martin, R. L.; Bishop, A. R. Molecular Geometry Fluctuation Model for the Mobility of Conjugated Polymers. Phys. Rev. Lett. 2000, 84, 721-724.

(59) Grozema, F. C.; van Duijnen, P. T.; Berlin, Y. A.; Ratner, M. A.; Siebbeles, L. D. A. Intramolecular Charge Transport along Isolated Chains of Conjugated Polymers: Effect of Torsional Disorder and Polymerization Defects. J. Phys. Chem. B 2002, 106, 7791-7795.

(60) Sprick, R. S.; Hoyos, M.; Morrison, J. J.; Grace, I. M.; Lambert, C.; Navarro, O.; Turner, M. L. Triarylamine polymers of bridged phenylenes by (N-heterocyclic carbene)-palladium catalysed C-N coupling. J. Mater. Chem. C 2013, 1, 3327-3336.

(61) Ma, B. C.; Ghasimi, S.; Landfester, K.; Vilela, F.; Zhang, K. A. I. Conjugated Microporous Polymer Nanoparticles with Enhanced Dispersibility and Water Compatibility for Photocatalytic Applications. J. Mater. Chem. A 2015, 3, 16064-16071.

(62) Airaksinen, S.; Karjalainen, M.; Shevchenko, A.; Westermarck, S.; Leppänen, E.; Rantanen, J.; Yliruusi, J. Role of Water in the Physical Stability of Solid Dosage Formulations. J. Pharm. Sci. 2005, 94, 2147-2165. 